\title{
Mechanism of Baicalein in the treatment of arthritis by regulating JNK/ERK/p38MAPK and PI3K/Akt signaling pathways
}

\author{
Xiaobo WANG ${ }^{1}$, Jing YANG ${ }^{1}$, Hongguo LIU², Jiandong LIU ${ }^{2 *}$, Lianjia WANG ${ }^{2 *}$
}

\begin{abstract}
The present study aimed to investigate the anti-inflammatory effects of Baicalein on human osteoarthritic chondrocytes and its molecular mechanisms, and to explore the related molecular events that may occur. The extracted human osteoarthritic chondrocytes were stimulated with interleukins. After administered with different concentrations of Baicalein, the survival rate of chondrocytes was observed. Apoptosis was detected by apoptosis assay kit. The effect on NO production was detected using the Griess reagent kit. The expressions of IL- 6 and PGE2 were detected by ELISA. The apoptotic proteins and PI3/Akt, NF-kB and MAPK cascade proteins were detected by WB assay. Baicalein significantly increased the survival rate of chondrocytes and decreased the expressions of NO, IL-6 and PGE2. The expressions of COX-2 and induced iNOS were significantly reduced in a dose-dependent manner under the administration of Baicalein. In addition, Baicalein significantly reduced the NF- $\kappa \mathrm{B}$ signaling pathway, inhibited the activation of the induced PI3/Akt, JNK, ERK and p38 MAPK cascades. Inflammatory mediators play a key role in the pathogenesis of osteoarthritis. Baicalin significantly inhibits the expression of inflammatory mediators in chondrocytes of human osteoarthritis and regulates the JNK/ERK/p38 MAPK and PBK/Akt signaling cascades. The experiment evaluated the anti-inflammatory and anti-osteomyelitis effects of Baicalein in order to discover new treatment strategies.
\end{abstract}

Keywords: baicalein; JNK/ERK/p38MAPK; PI3K/Akt; arthritis.

Practical Application: Baicalein in the treatment of arthritis.

\section{Introduction}

Osteoarthritis is a degenerative disease of the joint characterized by progressive damage and erosion of the articular cartilage, destruction of the articular surface, exposure of the subchondral bone, and long-term wear and tear, secondary bone hyperplasia, formation of callus and cartilage lower bone sclerosis, joint deformity, chronic pain, limited mobility, and reduced quality of life, which will eventually lead to long-term illness and disability (Riddle \& Stratford, 2014; Kato et al., 2014). The earliest lesions of osteoarthritis occur in articular cartilage. Under normal conditions, articular chondrocytes maintain a dynamic balance between the synthesis and degradation of extracellular matrix (ECM) components, including n-type collagen and proteoglycans in articular cartilage, the most abundant proteoglycan (PG) (Wojdasiewicz et al., 2014; Yang et al., 2014).

Due to the excessive production of inflammatory cytokines and matrix degrading enzymes, the chondrocyte metabolism is imbalanced, and synergistic with the decrease of anabolic signals, eventually leading to the destruction of extracellular matrix and consequent cartilage degeneration. Reactive oxygen species (ROS)-induced oxidative stress further disrupts cartilage homeostasis and promotes catabolism through inducing apoptosis and matrix component destruction, up-regulating potential matrix degrading enzymes, and inhibiting matrix synthesis as well as intracellular and cellular oxidation of external molecules (Tuli et al., 2020).
Baicalein mainly exists in Scutellaria Baicalensis Georgi, which has the effects of reducing cerebral vascular resistance, improving cerebral blood circulation, increasing cerebral blood flow and anti-platelet aggregation. At present, there are pre-clinical reports about the protective effects of Baicalein on bone, such as bone healing, but there are few reports of the drug effect on osteoarthritis, so this direction has a certain degree of innovation (Richette et al., 2010).

\section{Methods}

\subsection{Chondrocyte separation and cell culture}

The experiment was approved by the hospital ethics committee (No.: 20B1K0J50). Twenty five informed and consenting patients underwent total knee arthroplasty, with a definitive diagnosis of osteoarthritis/osteodystrophy and exclusion of other diseases.

Diagnostic criteria for knee osteoarthritis refer to the American College of Rheumatology diagnostic criteria for knee osteoarthritis/osteodystrophy.Articular cartilage is obtained from the primary cartilage cells acquired from the knee joint in patients with knee osteoarthritis. The cartilage tissue was cut into pieces, and digested with $0.25 \%$ trypsin for $30 \mathrm{~min}$, and then digested with $0.2 \%$ collagenase II and DMEM at $37^{\circ} \mathrm{C}$ for 6 hours. The cells were resuspended in DMEM containing $10 \%$ 
bovine serum albumin, $100 \mathrm{U} / \mathrm{mL}$ penicillin and $100 \mathrm{mg} / \mathrm{mL}$ streptomycin. The culture was carried out at $37^{\circ} \mathrm{C}$, and was simultaneously filled with $5 \%$ carbon dioxide (CO2).

\subsection{MTT assay detecting cell viability}

The obtained chondrocytes were seeded in a 96-well plate at a density of $6 \times 10^{3}$ per well and cultured overnight, and then the cells were subjected to different concentrations of Baicalein for 24 hours in Experiment 1: $0 \mu \mathrm{g} / \mathrm{mL}, 10 \mu \mathrm{g} / \mathrm{mL}, 20 \mu \mathrm{g} / \mathrm{mL}$, $40 \mu \mathrm{g} / \mathrm{mL}$ and $80 \mu \mathrm{g} / \mathrm{mL}$.

In Experiment 2, the cells were divided into five groups. A, the Control group, the cells were treated without Baicalein or IL- $1 \beta$. B, the Model group, the cells were treated with $5 \mathrm{ng} / \mathrm{mL}$ IL-l for 24 hours. C, the low concentration group, the cells were treated with $10 \mu \mathrm{g} / \mathrm{mL}$ Baicalein for 4 hours, then with $5 \mathrm{ng} / \mathrm{mL}$ IL-l for 24 hours. D, the medium concentration group, the cells were treated with $20 \mu \mathrm{g} / \mathrm{mL}$ Baicalein for 4 hours, then with $5 \mathrm{ng} / \mathrm{mL}$ IL-1 for 24 hours. E, the high concentration group, the cells were treated with $40 \mu \mathrm{g} / \mathrm{mL}$ Baicalein for 4 hours, then with $5 \mathrm{ng} / \mathrm{mL}$ IL-l for 24 hours.

The medium was removed, $20 \mathrm{~mL}$ of MTT $(5 \mathrm{mg} / \mathrm{mL})$ was added to each well for $4 \mathrm{~h}$ incubation. The supernatant was discarded and $150 \mu \mathrm{L}$ of dimethyl sulfoxide (DMSO) was added to dissolve the formazan crystals. The absorbance at $570 \mathrm{~nm}$ was measured by a microplate reader(Bio-Rad,Hercules,CA,USA).

\subsection{Annexin $V$ staining to detect apoptosis}

The cells were washed once with PBS and then digested with trypsin. After being washed with ice-cold PBS, the cells were resuspended in staining buffer. An appropriate amount of FITC-Annexin V staining solution was added according to the kit instructions. The cells were incubated in darkness.

\subsection{Detection of interleukin (IL-6) and prostaglandin E2 (PGE2)}

Chondrocytes were pretreated with different concentrations of Baicalein for 4 hours and then with IL-1 for 24 hours. The concentrations of IL-6 (MSK,NO.:69-23047) and PGE2(MSK,NO.:69-80118) in the supernatant were examined by enzyme-linked immunosorbent assay.

\subsection{Detection experiment of nitric oxide (NO)}

The concentrations of NO in the supernatant of chondrocytes treated with Baicalein and IL-1 at various concentrations of $10 \mu \mathrm{g} / \mathrm{mL}, 20 \mu \mathrm{g} / \mathrm{mL}$, and $40 \mu \mathrm{g} / \mathrm{mL}$ described above were detected using a Griess kit.

\subsection{Western Blot detecting contents in apoptotic proteins and PI3/Akt, NF-kB and MAPK cascades}

The cells were collected from each group, and washed twice with PBS. Each bottle was added with $400 \mu$ l of cell lysate, then with $40 \mu \mathrm{l}$ of $10 \mathrm{mmol} / \mathrm{L}$ PMSF. The bottles were gently agitated, then placed on ice for $10 \mathrm{~min}$ to lyse the cells sufficiently. The cells were repeatedly aspirated with a sterile syringe. Following this, the lysed product was added to an EP tube, and the EP tube was ice-bathed for $30 \mathrm{~min}$, then centrifuged at $12000 \mathrm{~g}$ for $15 \mathrm{~min}$. The supernatant was transferred to a new EP tube, and the protein concentration was quantified by a protein standard BCA method. After this, $100 \mu \mathrm{l}$ of each tube was added with $20 \mu \mathrm{l}$ of protein $6 \times$ Buffer and boiled for $5 \mathrm{~min}$, then stored at $-80^{\circ} \mathrm{C}$ for later use. The above samples were obtained, and proteins were separated by $12 \%$ SDS-PAGE electrophoresis, and the separated protein bands were transferred to PVDF membrane. The cells were blocked at room temperature for $1 \mathrm{~h}$, then incubated with the primary antibody (concentration 1:1000) at $4{ }^{\circ} \mathrm{C}$ overnight. The cells were washed with PBST three times. The secondary antibody (1:1000) was added for $1 \mathrm{~h}$ incubation. The cells were washed with PBST three times. Color development and fixation were conducted by chemiluminescence. The expressions of each of the above proteins were determined.

\subsection{Statistical approach}

All data are expressed as mean \pm standard deviation (mean \pm SD). The data has passed the normal distribution assessment. The $\mathrm{t}$-test was used for comparison between the two groups, and the comparison between multiple groups of data $(>2)$ was performed using One-way ANOVA. There was a statistical significance at $p<0.05$. The data was analyzed using Graphpad Prism 5.0 (Graphpad Software. San Diego, CA).

In reference to the "Diagnostic Criteria for Knee Osteoarthritis" of the American Academy of Rheumatology (ARA), the calculation for the sample size is selected in this study.

The clinical diagnostic criteria are as follows:

(1) Recurrence of knee joint pain in the past month;

(2) There is a bone friction sound when the knee joint moves;

(3) Morning stiffness of the knee joint less than $30 \mathrm{~min}$;

(4) Hypertrophy of the knee joint with bone hyperplasia;

(5) Age more than 40.

Those who meet the above (1), (2), (3) and (4) or (1), (2), (3) and (5) can be clinically diagnosed as knee osteoarthritis.

The combined clinical and radiology diagnostic criteria are as follows:

(1) Knee pain most of the time in the past month;

(2) X-ray shows the formation of osteophytes on the edge of the knee joint;

(3) Synovial fluid laboratory examination accords with osteoarthritis;

(4) Age $\leq 40$;

(5) Morning stiffness of the knee joint $<30$ minutes;

(6) There is bone friction sound when the joints move.

Those who meet (1) and (2) or (1), (3), (5) and (6) or (1), (4), (5) and (6) can be diagnosed as knee osteoarthritis. 


\section{Results}

\subsection{The effect of Baicalein on cell viability}

The chondrocytes treated with different concentrations of Baicalein were tested by MTT assay to detect whether the drug has cytotoxic effect. It is found that the viability of chondrocytes was almost unaffected regardless of the concentration of drug used, as shown in Figure 1. The results showed that Baicalein did not have a cytotoxic effect. After IL-1 $\beta$ stimulation, it can be seen that Baicalein improved the survival rate of chondrocytes, as shown in Figure 2.

\subsection{Baicalein inhibited the production of NO and PGE2 induced by IL-1 $\beta$ stimulation}

The results displayed that the production of NO and PGE2 increased significantly after chondrocytes were stimulated with IL- $1 \beta$. Besides, the content of inflammatory cytokine IL- 6 was also elevated. However, after treatment with Baicalein, the contents

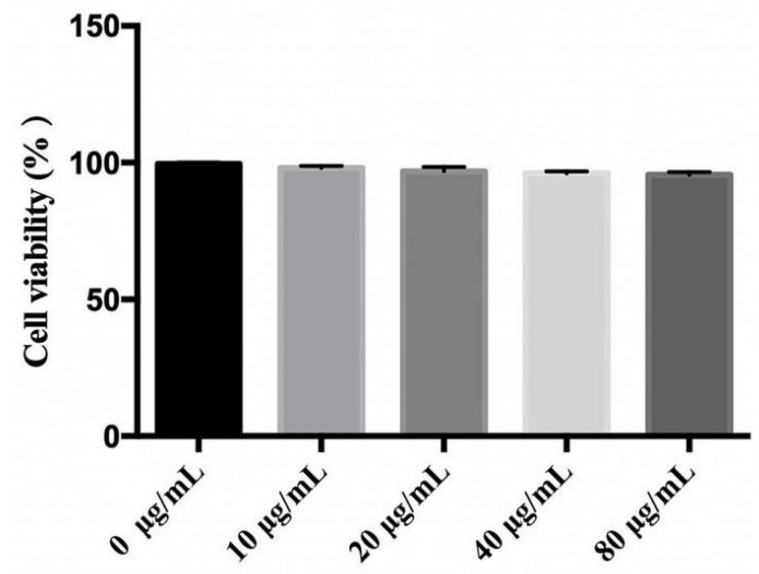

Figure 1. Effect of Baicalein on cell viability.

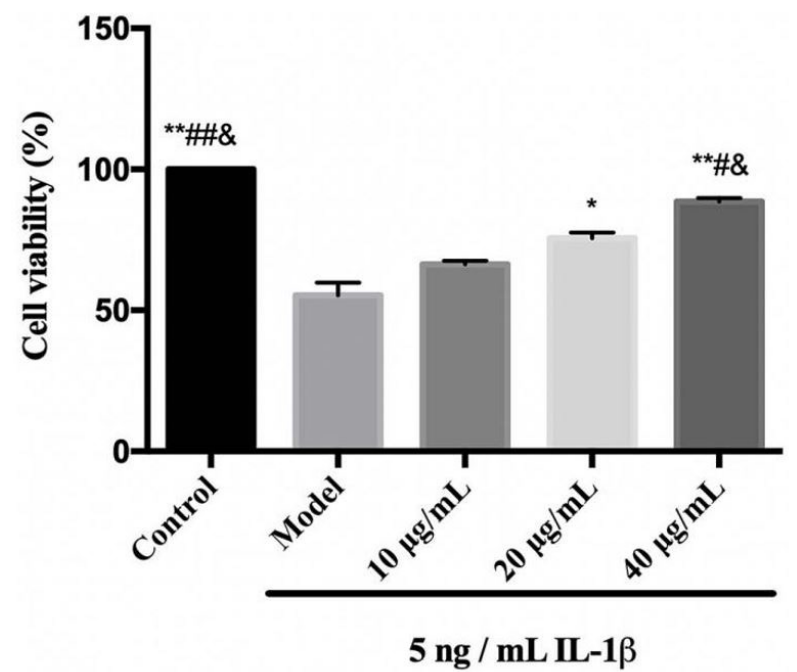

Figure 2. Effect of Baicalein on cell viability. of NO and PGE2 were significantly decreased, suggesting that Baicalein is effectively anti-inflammatory, and the higher the concentration, the better the effect, as shown in Figure 3.

\subsection{Western Blot detecting the contents of apoptotic proteins, as well as proteins in PI $3 \mathrm{~K} / \mathrm{Akt}, \mathrm{NF- \kappa B}$ and MAPK cascades}

The results showed that the expressions of proapoptotic proteins Bax and Bad increased in chondrocytes treated with IL-1 $\beta$, while the expressions of anti-apoptotic proteins Bcl-2 and Bcl-xL decreased (Figure 4). The increase in the number of apoptosis may be due to an increase in the expression of pro-apoptotic proteins. The expressions of $\mathrm{Bcl}-2$ and $\mathrm{Bcl}-\mathrm{xL}$ were significantly elevated after the administration of Baicalein. At the same time, the expressions of Bax and Bad were significantly decreased. The above results indicated that Baicalein has an effect on the expressions of Bax, Bad, Bcl-2 and Bcl-xL in the apoptotic pathway, suggesting an anti-apoptotic effect.

Stimulation of chondrocytes by IL- $1 \beta$ significantly elevated the expressions of JNK, ERK and p38 MAPK (Figure 5). The treatment of Baicalein significantly decreased the expressions of JNK, ERK and p38MAPK. Phosphorylated JNK and p38 MAPK decreased more significantly than p-ERK. The expression of ERK also decreased under the administration of Baicalein, but the decrease was not as obvious as that of JNK and p38 MAPK. After the chondrocytes were treated with IL- $1 \beta$, the expressions of p-NF- $\kappa B, p 65, p-I \kappa B \alpha$ and TNF- $\alpha$ were significantly increased. However, after administration with Baicalein, the expressions of the above proteins were significantly reduced (Figure 6), indicating that the decrease of NF- $\mathrm{kB}$ signaling may lead to a decrease in the expression of inflammatory mediators, thereby reducing the inflammatory response. When chondrocytes were stimulated with IL-1 $\beta$, high concentrations of Akt, phosphorylated Akt, phosphorylated GSK-3P and mTOR were produced in the cells, while PTEN expression was lower. However, after treatment with Baicalein, the expressions of phosphorylated Akt, GSK-3P and mTOR were significantly inhibited (Figure 7). In addition, Baicalein can elevate the expression of PTEN in a dose-dependent manner. Therefore, Baicalein can effectively regulate the PI3K/Akt signaling pathway, which also partially caused the reduce of NF- $\mathrm{kB}$ expression.

\subsection{Effect of Baicalein on apoptosis}

Annexin V / PI double staining showed that apoptosis was obvious in the control group, but it was significantly inhibited in the drug group, as shown in Figure 8.

\section{Discussions}

The NF- $\mathrm{kB}$ and MAPK signaling pathways play a crucial role in the regulation of inflammatory mediators (Li et al., 2011). Under physiological and pathological conditions, NF- $\kappa B$ can regulate various genes involved in immune function and inflammation. Inflammation is a reaction mechanism of the body against pathogen invasion. (Wang \& Dong, 2021) It is not a simple but a complex biological process.F- $\kappa B$ is involved in the inflammatory response, and regulates many molecules involved in the early immune response and various stages of the inflammatory 

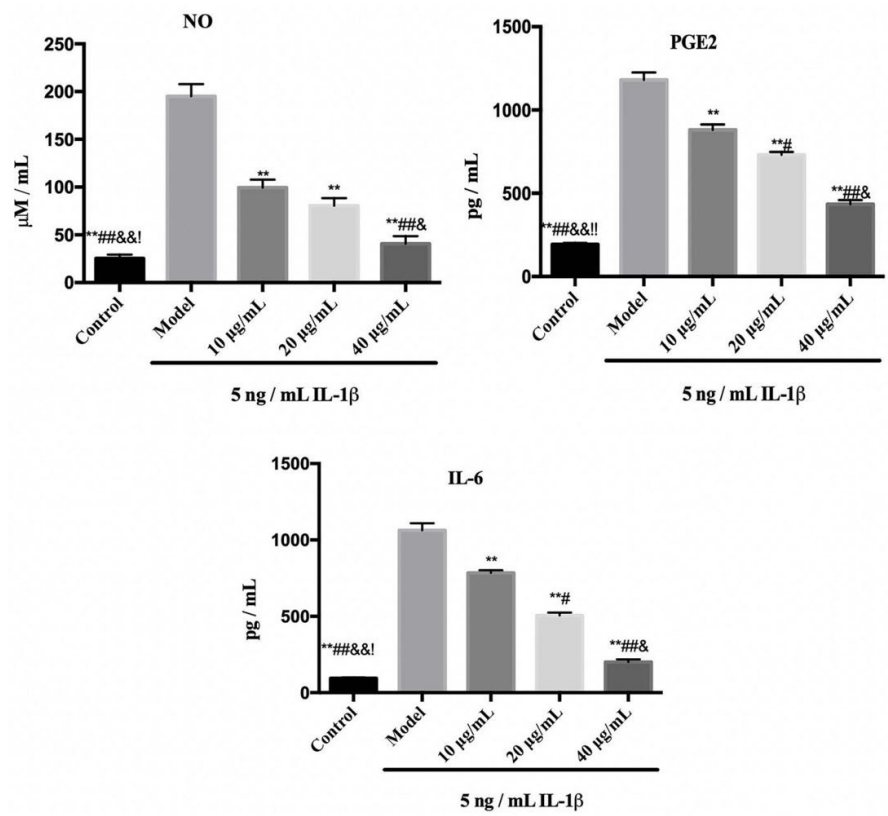

Figure 3. Baicalein inhibiting production of NO and PGE2 induced by IL- $1 \beta$ stimulation.
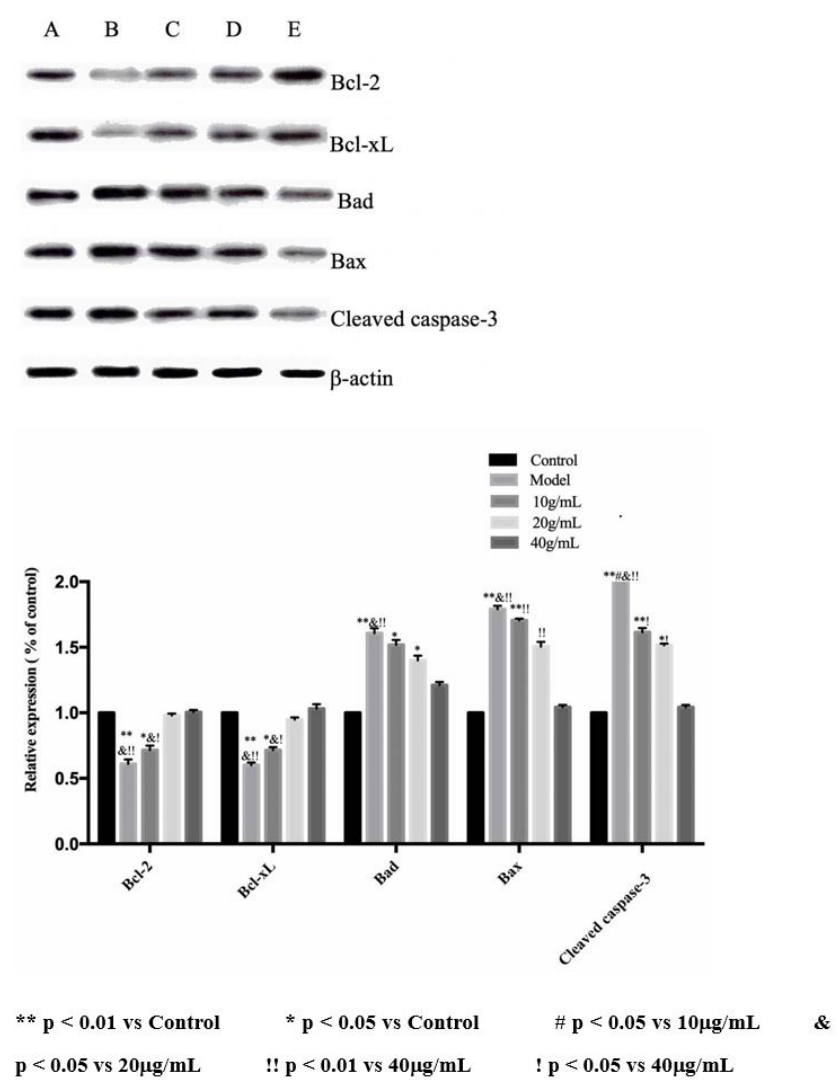

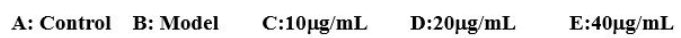

Figure 4. Effect of drugs on various apoptotic proteins.

response. It is highly active in the inflammatory sites of various inflammatory diseases (Zhu \& Liu, 2020). NF- $\kappa$ B can efficiently induce inflammatory cytokines (IL-1, IL-6, and TNF- $\alpha$ ), adhesion molecules (ICAM-1 and VCAM-1), inflammatory enzymes
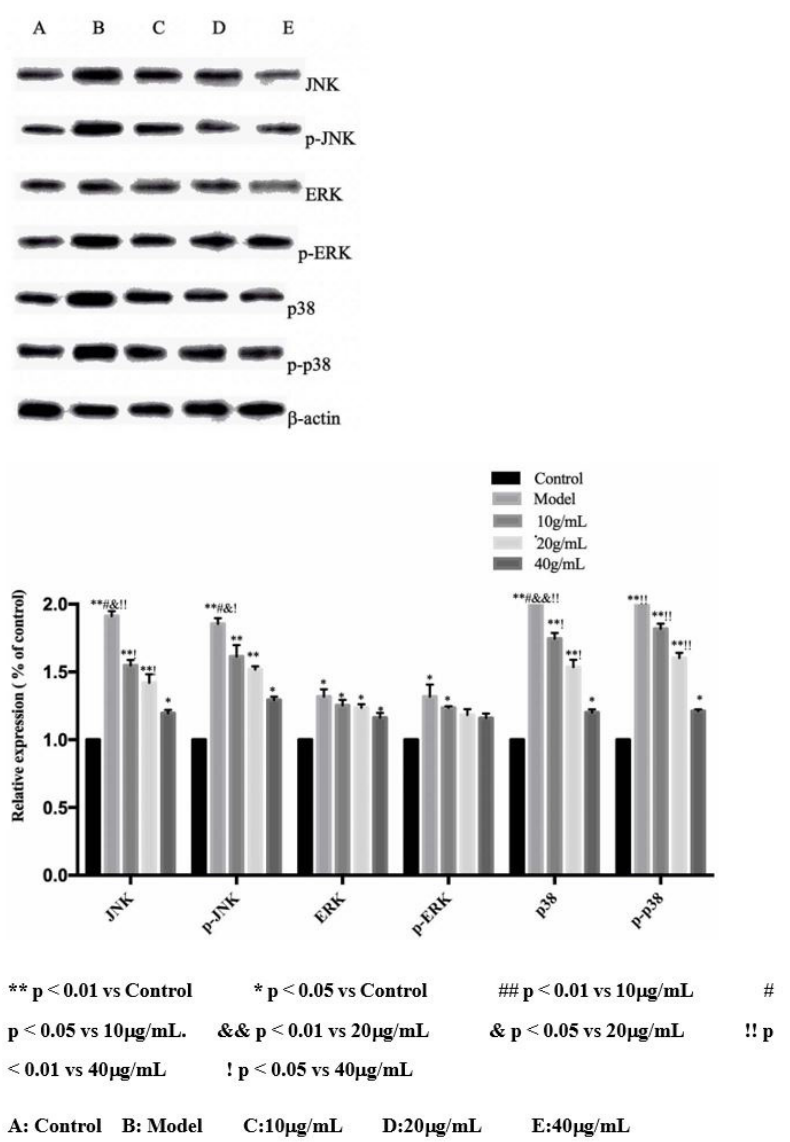

Figure 5. Effect of drugs on various proteins in the MAPK cascade.

(iNOS and COX-2), and chemokines, etc. NF- $\kappa$ B amplifies the inflammatory cascade, making it a transcription factor of many inflammatory cytokines in inflammation (Kato et al., 2014; Sun et al., 2017). 

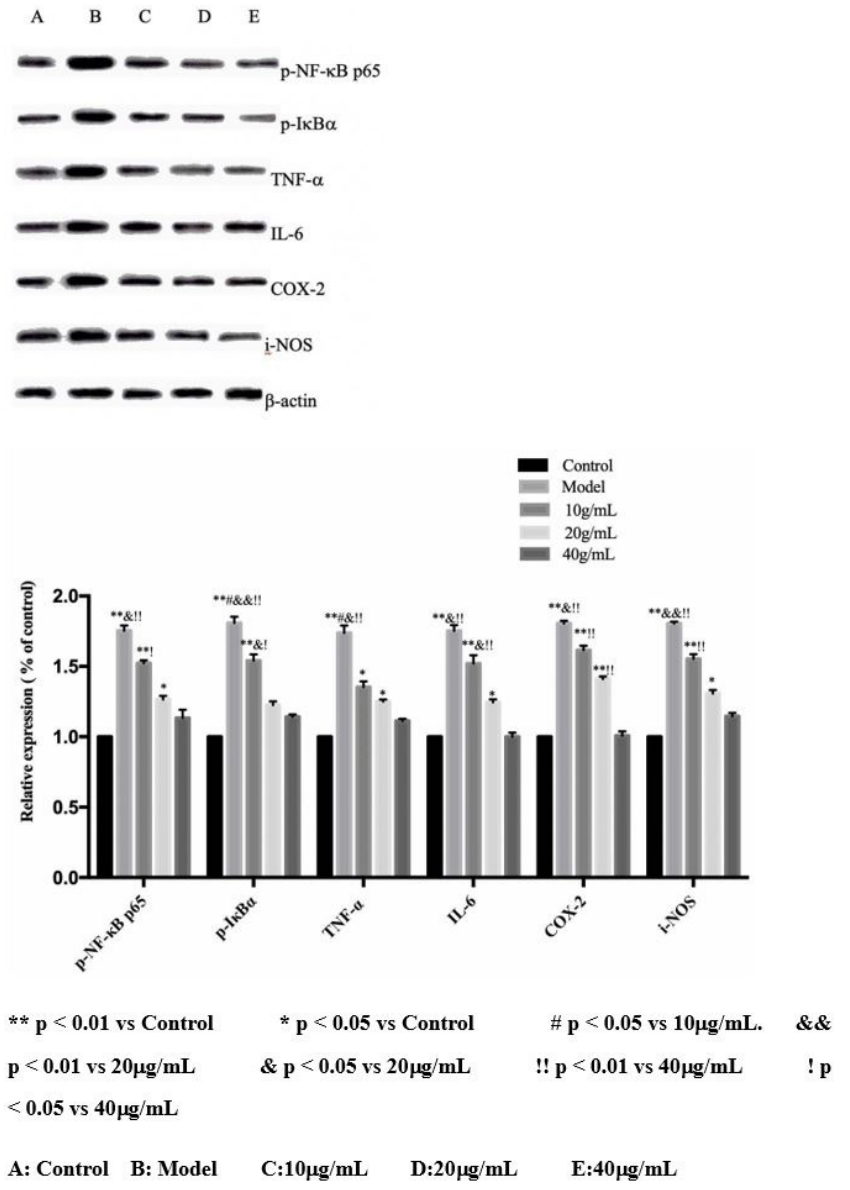

Figure 6. Effect of drugs on NF- $\kappa B$ signaling pathway.

The mitogen-activated protein kinase (MAPK) signaling pathway plays an important role in the regulation of inflammatory mediators (Fu et al., 2016; Akhtar et al., 2017). P38 is the most important member of the MAPK family to control inflammation, and its activation can be caused by lipopolysaccharide, physiological stress, ultraviolet radiation, and osmotic stress (Valdes \& Spector, 2011). Key enzymes of the P38 pathway include ASK, MLK, and TAK of the MAPK class, as well as MKK6 and MKK3 of the MAPKK class. TAK binding protein (TAB) activates TAK and mediates signal transduction of transforming growth factor. TAK also activates MKK4, which in turn activates P38 (Liu et al., 2020). Nuclear translocation occurs after P38 activation, causing phosphorylation and activation of many protein kinases and transcription factors (Jeong et al., 2016; Zhou et al., 2016; Wang et al., 2015).

The result of PI3K activation is the production of the plasma membrane. The two messengers PIP3 and HP3 bind to the $\mathrm{PH}$ domain-containing signaling proteins Akt and PDK1, and cause the phosphorylation of Ser308 of the Akt protein by PDK1, as well as the activation of Akt. Akt can also be activated by the phosphorylation of Thr473 by PDK2. The activated Akt activates or inhibits its downstream target proteins through phosphorylation of Bad, Caspase9, NF- $\kappa$ B, GSK-3, and FKHR, thus regulating cell proliferation, cell differentiation, apoptosis and cell migration. The product of the tumor suppressor gene
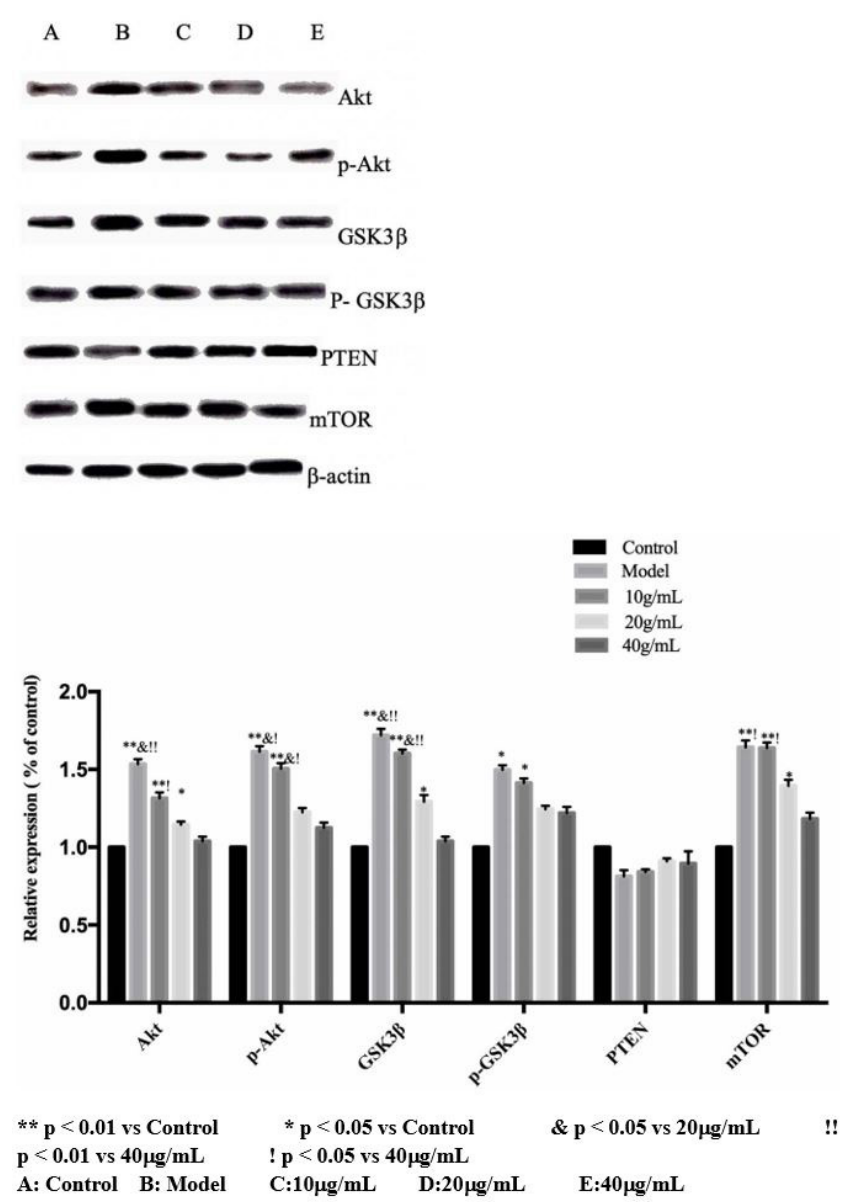

Figure 7. Effect of drugs on PI3/Akt signaling pathway.

PTEN induces dephosphorylation of inositol 3-phosphate and negatively regulates the PI3K pathway. PI3K and Akt are upstream molecules of the NF- $\kappa \mathrm{B}$ signaling pathway and play a crucial role in the activation of NF- $\mathrm{BB}$ (Yang et al., 2013; Wang et al., 2014)). When chondrocytes were stimulated with IL-1 $\beta$, high concentrations of Akt, phosphorylated Akt, phosphorylated GSK-3P and mTOR were produced in the cells, while PTEN expression was lower. However, after treatment with Baicalein, the expressions of phosphorylated Akt, GSK-3P and mTOR were significantly inhibited (Figure 7). In addition, Baicalein can elevate the expression of PTEN in a dose-dependent manner. Therefore, Baicalein can effectively regulate the PI3K/Akt signaling pathway, which also partially caused the reduce of NF- $\kappa B$ expression.

To investigate the effect of Baicalein on PI3K/Akt signaling, the expression of proteins in the above pathways were examined. IL-1 $\beta$ can induce several times the expression of phosphorylated Akt and GSK-3 $\beta$, indicating that it can activate the pathway. The pretreatment of Baicalein significantly inhibited this pathway, showing a significant decrease in the phosphorylation level of Akt. These results suggest that the inhibition of NF- $\kappa \mathrm{B}$ activation by Baicalein may be involved in the PBK/Akt cascade. After the chondrocytes were treated with IL-1 $\beta$, it was found that the expressions of related proteins JNK, ERK and p38 in the MAPK pathway increased, while Baicalein inhibited the expressions of 
Control
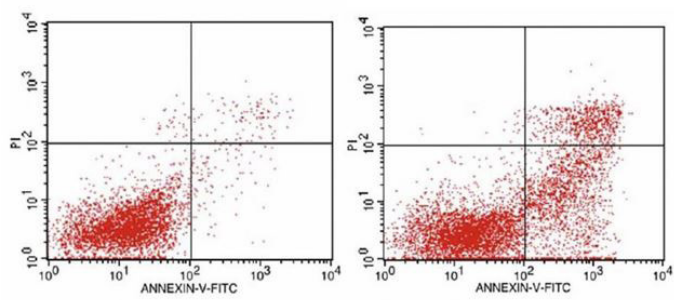

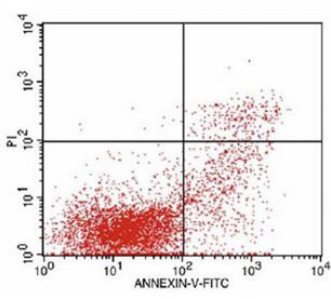

$10 \mu \mathrm{g} / \mathrm{mL}$

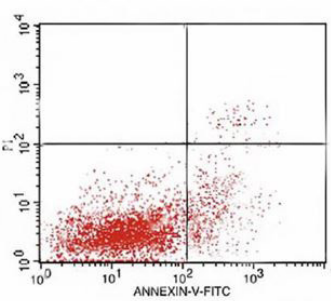

$20 \mu \mathrm{g} / \mathrm{mL}$

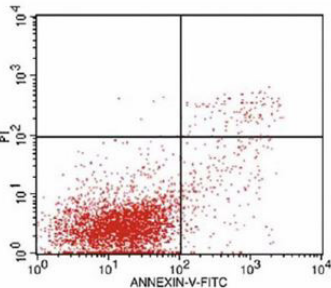

$40 \mu \mathrm{g} / \mathrm{mL}$

\section{** p $<0.01$ vs Control \\ p $<0.05$ vs $20 \mu \mathrm{g} / \mathrm{mL}$}

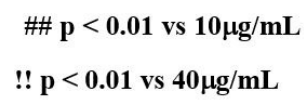

\&

! p $<0.05$ vs $40 \mu \mathrm{g} / \mathrm{mL}$

Figure 8. Effect of Baicalein on apoptosis.

JNK, ERK and p38 MAPK, indicating that Baicalein can inhibit the activation of MAPK. There was no significant change in the expression of ERK compared to changes in the expressions of JNK and p38 MAPK, although some significant changes were observed. Baicalein can effectively inhibit the MAPK pathway in chondrocytes treated with IL-1 $\beta$, which may partly explain the observed decrease in inflammatory mediator levels.

\section{Conclusion}

Although great research work and efforts have been made in recent years, the treatment of osteoarthritis is still experimental and unknown. There is a wide range of interaction among various cytokines to maintain cartilage metabolism balance in vivo. Any drug can not affect a single cytokine, and it is impossible to completely cause complete cartilage repair or affect arthritis joint in clinic. On the contrary, joint interaction is needed.

In summary, inflammatory mediators play a key role in the pathogenesis of osteoarthritis. Despite tremendous research efforts in recent years, the treatment of osteoarthritis is still experimental and unknown. There are a wide range of interactions between various cytokines that maintain the balance of cartilage metabolism in the body. It is impossible for any drug to affect a single cytokine, it is even less likely to cause complete cartilage repair or clinically affect arthritic joints. Baicalein can significantly inhibit the expression of inflammatory mediators in human osteoarthritic chondrocytes and regulate the JNK/ERK/p38 MAPK and PBK/Akt signaling cascades.

\section{Ethical approval}

This study is approved by the Ethics Committee of Wendeng Orthopedic and Traumatic Hospital.

\section{Conflicts of interest}

None.

\section{Availability of data and material}

The datasets used or analysed during the current study are available from the corresponding author on reasonable request.

\section{Author contribution}

Xiaobo Wang contributed to research concept and design. Jing Yang contributed to collection and/or assembly of data. Hongguo Liu contributed to data analysis and interpretation. Jiandong Liu contributed to writing the article. Lianjia Wang contributed to critical revision of the article. Lianjia Wang and Jiandong Liu helped to the final approval of the article.

\section{References}

Akhtar, N., Khan, N. M., Ashruf, O. S., \& Haqqi, T. M. (2017). Inhibition of cartilage degradation and suppression of PGE (2) and MMPs expression by pomegranate fruit extract in a model of posttraumatic osteoarthritis. Nutrition, 33(8), 1-13. http://dx.doi.org/10.1016/j. nut.2016.08.004. PMid:27908544.

Fu, Y., Lei, J., Zhuang, Y., Zhang, K., \& Lu, D. (2016). Overexpression of HMGB1 A-box reduced IL-Ib induced MMP expression and the 
production of inflammatory mediators in human chondrocytes. Experimental Cell Research, 349(1), 184-190. http://dx.doi.org/10.1016/j. yexcr.2016.10.014. PMid:27771306.

Jeong, J. W., Lee, H. H., Lee, K. W., Kim, K. Y., Kim, S. G., Hong, S. H., Kim, G. Y., Park, C., Kim, H. K., Choi, Y. W., \& Choi, Y. H. (2016). Mori folium inhibits interleukin-lp-induced expression of matrix metailoproteinases and inflammatory mediators by suppressing the activation of NF-kB and p38 MAPK in SW1353 human chondrocytes. International Journal of Molecular Medicine, 37(2), 452-460. http:// dx.doi.org/10.3892/ijmm.2015.2443. PMid:26707272.

Kato, T., Miyaki, S., Ishitobi, H., Nakamura, Y., Nakasa, T., Lotz, M. K., \& Ochi, M. (2014). Exosomes from IL-lbeta stimulated synovial fibroblasts induce osteoarthritic changes in articular chondrocytes. Arthritis Research \& Therapy, 16(4), R163. http://dx.doi.org/10.1186/ ar4679. PMid:25092378.

Li, X., Kim, J. S., van Wijnen, A. J., \& Im, H. J. (2011). Osteoarfliritic tissues modulate functional properties of sensory neurons associated with symptomatic OA pain. Molecular Biology Reports, 38(8), 53355339. http://dx.doi.org/10.1007/s11033-011-0684-7. PMid:21327824.

Liu, J. L., Zeng, X. X., Lin, Q. Z., He, W. F., \& Yang, Q. C. (2020) The benefits and harms of Chinese medicinal herbs for the treatment of rapidly progressive glomerulonephritis in adult patients. Food Science and Technology. In press. https://doi.org/10.1590/fst.23620.

Richette, P., Poitou, C., Garnero, P., Vicaut, E., Bouillot, J. L., Lacorte, J. M., Basdevant, A., Clément, K., Bardin, T., \& Chevalier, X. (2010). Benefits of massive weight loss on symptoms, systemic inflammation and cartilage turnover in obese patients with knee osteoarthritis. Annals of the Rheumatic Diseases, 70(1), 139-144. http://dx.doi. org/10.1136/ard.2010.134015. PMid:20980288.

Riddle, D. L., \& Stratford, P. W. (2014). Knee pain during daily tasks, knee osteoarthritis severity, and wide spread pain. Physical Therapy, 94(4), 490-498. http://dx.doi.org/10.2522/ptj.20130331. PMid:24231230.

Sun, H. Y., Hu, K. Z., \& Yin, Z. S. (2017). Inhibition of the p38-MAPK signaling pathway suppresses the apoptosis and expression of proinflammatory cytokines in human osteoarthritis chondrocytes. Cytokine, 90, 135-143. http://dx.doi.org/10.1016/j.cyto.2016.11.002. PMid:27907835.

Tuli, H. S., Aggarwal, V., Kaur, J., Aggarwal, D., Parashar, G., Parashar, N. C., Tuorkey, M., Kaur, G., Savla, R., Sak, K., \& Kumar, M. (2020).
Baicalein: A metabolite with promising antineoplastic activity. Life Sciences, 259, 118183.

Valdes, A. M., \& Spector, T. D. (2011). Genetic epidemiology of hip and knee osteoarthritis. Nature Reviews. Rheumatology, 7(1), 2332. http://dx.doi.org/10.1038/nrrheum.2010.191. PMid:21079645.

Wang, J., \& Dong, J. (2021). Study on the mechanism and therapeutic effect of Antelope horn treatment on cerebral ischemia reperfusion injury of mouse. Food Science and Technology, 41(Suppl. 1), 142-149. https://doi.org/10.1590/fst.35119.

Wang, L., Ma, T., \& Zheng, Y. (2015). Diosgenin inhibits IL-lb -induced expression of inflammatory mediators in human osteoarthritis chondrocytes. International Journal of Clinical and Experimental Pathology, 8(5):4830-6.

Wang, L., Xu, Y., Yu, Q., Sun, Q., Xu, Y., Gu, Q., \& Xu, X. (2014). H-RN, a novel anti-angiogenic peptide derived from hepatocyte growth factor inhibits inflammation in-vitro and in-vivo through PI3K7AKT/IKK/ NF-kappa B signal pathway. Biochemical Pharmacology, 89(2), 255265. http://dx.doi.org/10.1016/j.bcp.2014.02.026. PMid:24630926.

Wojdasiewicz, P., Poniatowski, L. A., \& Szukiewicz, D. (2014). The role of inflammatory and anti-inflammatory cytokines in the pathogenesis of osteoarthritis. Mediators of Inflammation, 2014(3), 561459. http:// dx.doi.org/10.1155/2014/561459. PMid:24876674.

Yang, B., Kang, X., Xing, Y., Dou, C., Kang, F., Li, J., Quan, Y., \& Dong, S. (2014). Effect of microRNA-145 on E.-lbeta-induced cartilage degradation in human chondrocytes. FEBS Letters, 588(14), 23442352. http://dx.doi.org/10.1016/j.febslet.2014.05.033. PMid:24873879.

Yang, P., Han, Y., Gui, L., Sun, J., Chen, Y. L., Song, R., Guo, J. Z., Xie, Y. N., Lu, D., \& Sun, L. (2013). Gastrodin attenuation of the inflammatory response in $\mathrm{H} 9 \mathrm{c} 2$ cardiomyocytes involves inhibition of NF-kappaB and MAPKs activation via the phosphatidylinositol 3-kinase signaling. Biochemical Pharmacology, 85(8), 1124-1133. http://dx.doi.org/10.1016/j.bcp.2013.01.020. PMid:23376120.

Zhou, K., Hu, L., Liao, W., Yin, D., \& Rui, F. (2016). Coptisine Prevented IL-b Induced Expression of Inflammatory Mediators in Chondrocytes. Inflammation, 39(4), 1558-1565. http://dx.doi.org/10.1007/s10753016-0391-6. PMid:27294276.

Zhu, Y. X., \& Liu, G. (2020). The relationship between the insertion/ deletion gene polymorphism of ACE and atrial fibrillation in Chinese population: a meta-analysis. Food Science and Technology, In press. https://doi.org/10.1590/fst.36020. 